\title{
Performance of a quick pregnancy test on whole blood in early pregnancy units: a prospective cohort study Caroline Legoupil ${ }^{\mathrm{a}, \mathrm{c}}$, Isabelle Enderle ${ }^{\mathrm{a}, \mathrm{c}}$, Flore-Anne Le Baccon ${ }^{\mathrm{a}, \mathrm{c}}$, Claude Bendavid ${ }^{b, c}$, Lucas Peltier ${ }^{b, c}$, Estelle Bauville ${ }^{a}$, Jean Leveque ${ }^{a, c, d}$, Vincent Lavoue ${ }^{\mathrm{a}, \mathrm{c}, \mathrm{d}}$ and Maela Le Lous ${ }^{\mathrm{a}, \mathrm{c}}$
}

Objective To assess the diagnostic performance of the NG-Test human chorionic gonadotropin (hCG) WB, which is a new point-of-care (POC) hCG whole-blood test.

\begin{abstract}
Materials and methods This prospective study included women consulted in early pregnancy units for vaginal bleeding and/or pelvic pain with unknown pregnancy status after medical consultation including a pelvic ultrasound scan. A new POC test (the NG-Test hCG WB) and the usual laboratory serum test (considered the gold standard) were performed in patients. The results were interpreted in a blinded manner. Sensitivity, specificity, positive predictive value (PPV), and negative predictive value (NPV) were calculated for the NG-Test hCG WB.
\end{abstract}

Results During the study period, 200 patients were included. The pregnancy rate was $17 \%$. For the laboratory test, with a 5 UI/I hCG positivity threshold, the sensitivity, specificity, PPV, NPV, and Youden index of the NG-Test hCG WB were $89.7,100,100,97.9$, and $0.90 \%$, respectively. Considering a $10 \mathrm{UI/I}$ hCG positivity threshold, test sensitivity, specificity, PPV, NPV, and Youden index were $96.3,100,100,99.3$, and $0.96 \%$, respectively. False-negative cases were either extremely brief pregnancies or residual

\section{Introduction}

Pelvic pain (PP) and vaginal bleeding (VB) are present in $71-88 \%$ of women consulted in early pregnancy units $[1,2]$. The systematic management of these women relies on the identification of a pregnancy and its location [i.e. intrauterine pregnancy (IUP) or ectopic pregnancy (EP)]. Both physical examination and ultrasound usually help with the diagnosis. However, in approximately one-third of cases, pregnancy status cannot be established without a biological pregnancy test [1,2]. The gold-standard test for pregnancy is based on an immunologic dose of human chorionic gonadotropin (hCG) through a serum pregnancy test, with a $5 \mathrm{UI} / 1$ positivity threshold [3].

There has been increasing pressure to improve emergency department efficiency and throughput. Turnaround times for laboratory results have been shown to significantly influence throughput, with qualitative quick pregnancy testing often delaying advanced imaging, medication delivery, and disposition decisions. Usually, urinary tests are used, but they have shown a variable sensitivity with a theoretical detection threshold of hCG of 20-25 UI/l.
hCG after miscarriage. The result was obtained within 5 min with the NG-Test hCG WB versus $90 \pm 31 \mathrm{~min}$ with the laboratory test. It was easy to use.

Conclusion The NG-Test hCG WB showed a high sensitivity, specificity, PPV, and NPV. Its use as triage in the case of a negative pelvic ultrasound exam is a potential strategy to improve patient flow, with an average time saving of $85 \mathrm{~min}$.

Keywords: early pregnancy, human chorionic gonadotropin, point-of-care test, whole blood

Departments of a Gynecology, Obstetrics and Reproductive Medicine, ${ }^{b}$ Biochemistry, University Hospital of Rennes, ${ }^{\mathrm{c}}$ Faculty of Medicine, University of Rennes and INSERM U1242, Rennes, France

Correspondence to Maela Le Lous, MD, Department Gynecology, Obstetrics and Reproductive Medicine University Hospital of Rennes, 16 Bd Bulgarie, BP 90347, F-35 203 Rennes Cedex 2, France

Tel: + 3302992631 07; fax: + 330299266740 e-mail: maela.le.lous@chu-rennes.fr

Nevertheless, false-negative point-of-care (POG) urine pregnancy results are infrequent, but the large number of tests ordered in a high-volume emergency department can be expected to yield a significant cohort and thus increase the risk of missed EP [4].

A new POC qualitative pregnancy test that detects hCG in whole blood was developed recently, called the NGTest hCG WB (Laboratoire NG Biotech, Z.A Courbouton, Guipry, France). It works thanks to a simple blood drop (in the way diabetic patients perform glycemic control tests on their finger). It is easy to use during a medical consultation at any time and can be performed by a nurse. The positivity threshold is $10 \mathrm{UI} / 1$ and the results are obtained within $5 \mathrm{~min}$.

The main aim of this study was to assess the diagnostic performance of the NG-Test hCG WB. The secondary objectives were to assess the usefulness of performing the test, the cost-effectiveness, and the impact on throughput for women consulted in early pregnancy units for $\mathrm{PP}$ or $\mathrm{VB}$. 


\section{Materials and methods Population}

A prospective cohort study was carried out. A total of 200 women consulted in early pregnancy units at a tertiary hospital for PP and/or VB between 9 December 2016 and 3 December 2017 were included in the study. The sample size was calculated to detect more than 15 events (i.e. positive pregnancy tests). The inclusion criteria were the absence of a visible pregnancy with transvaginal ultrasound, at first medical contact, and the necessity to perform a pregnancy test. The exclusion criteria were patients younger than 18 years of age and patients who were treated for infertility.

All patients provided their consent after receiving oral and written information about the study. This study was approved by a local ethics committee (IRB, France Avis no. 16.84).

In our current protocol, patients consulted for $\mathrm{PP}$ or $\mathrm{VB}$ undergo a physical examination and an ultrasound scan (Philips HD7xe; Philips, Amsterdam, the Netherlands). If required, a quantitative hCG serum test is performed. Patients who were included in the study were also tested in a blinded manner using the NG-Test hCG WB at the end of the consultation. Patients included in the study were managed according to our standard policy on the basis of ultrasound findings and a serum pregnancy test. Medical management was not modified by the results of the NG-Test hCG WB.

The NG-Test hCG WB (Laboratoire NG Biotech) is a qualitative hCG whole-blood test based on an immunochromatographic technique. Therefore, hCG is recognized in its intact form by a monoclonal pair of antibodies: anti- $\alpha-h C G$ and anti- $\beta-h C G$. It was standardized according to the 5th International Standard for hCG of the National Institute for Biological Standards and Control NIBSG (preparation 07/364) [5]. The positivity threshold is $10 \mathrm{UI} / 1$. A nurse made a small finger incision with a lancing device and then dropped a small quantity of blood onto the test before adding the reactive. After $5 \mathrm{~min}$, the result was read by the nurse, who was blinded to the laboratory results. The result was considered positive if the test displayed two lines and negative if there was only one line (Fig. 1). If no line was visualized, the test was considered invalid and was repeated.

\section{Laboratory test}

All included patients had a laboratory test with a quantitative serum hCG measurement, which is considered the 'gold-standard' technique, using a venous blood sample drawn by a nurse. A dose of hCG was administered on isolated serum after centrifugation of the tubes with an electrochemiluminescence immunoassay using the $\mathrm{hCG}+\beta$ Roche reagent, which detects intact hCG and unity $\beta$, on the automate COBAS e411 (Roche Diagnostics GmbH, Mannheim, Germany). This method was standardized according to the 4th International Standard for hCG of the NIBSC (preparation 75/589) [6]. The inferior limit of detection was $0.1 \mathrm{UI} / 1$ and the positivity threshold was $5 \mathrm{UI} / 1$ for the gold-standard test. The results were read by technicians and validated by a biologist, who were all blinded to the NG-Test hCG WB results.

\section{Data collection}

The following data were recorded: patient age, gravidity, parity, date of last menstruation, contraception, smoking, clinical symptoms, physical examination data, ultrasound data, quick test result, gold-standard quantitative pregnancy test result, final diagnosis, and treatment received at patient discharge. PP was rated using a Numerical Pain Scale from 0 to 10 , with 0 corresponding to no pain and 10 representing the worst pain imaginable.

Nurses who were involved in the performance of the NG-Test hCG WB completed a questionnaire on the ease of use and reading of the result. They also had to report their overall satisfaction.

Finally, the time of patient arrival, time of blood sampling, time of result obtainment, and time of patient discharge were recorded.

\section{Outcome measure}

The main outcome was a positive laboratory pregnancy test. We also assessed the diagnostic performance of the NG-Test hCG WB at the threshold of $10 \mathrm{UI} / 1$ of hCG, which was the positivity threshold established by $\mathrm{NG}$ Biotech. Secondary outcomes included the global satisfaction of the nurses, the time between blood sampling and the result, and the costs.

\section{Statistical analysis}

Sensitivity (Se), specificity ( $\mathrm{Sp}$ ), positive predictive value (PPV), negative predictive value (NPV), and Youden index $(Y=\mathrm{Se}+\mathrm{Sp}-1)$ were calculated. Data were compared using a $\chi^{2}$-test and Fisher's test for qualitative variables, and a $t$-test for continuous variables. A $P$ value less than 0.05 was considered significant.

\section{Results Population}

Two hundred patients were included in the study. Twenty-seven (13.5\%) patients were excluded because of missing data and three other patients were excluded a posteriori because of mistaken inclusion: one patient had a confirmed EP and two other patients had an IUP diagnosed by ultrasound. A total of 170 patients were included in the analysis (Fig. 2). The characteristics of 170 patients are described in Table 1. Twenty-nine (17\%) patients had a pregnancy diagnosis confirmed by the goldstandard serum test. Age, smoking, gravidity, parity, and symptomatology (VB or pain) rates were similar in both groups (i.e. pregnant women vs. nonpregnant women). 

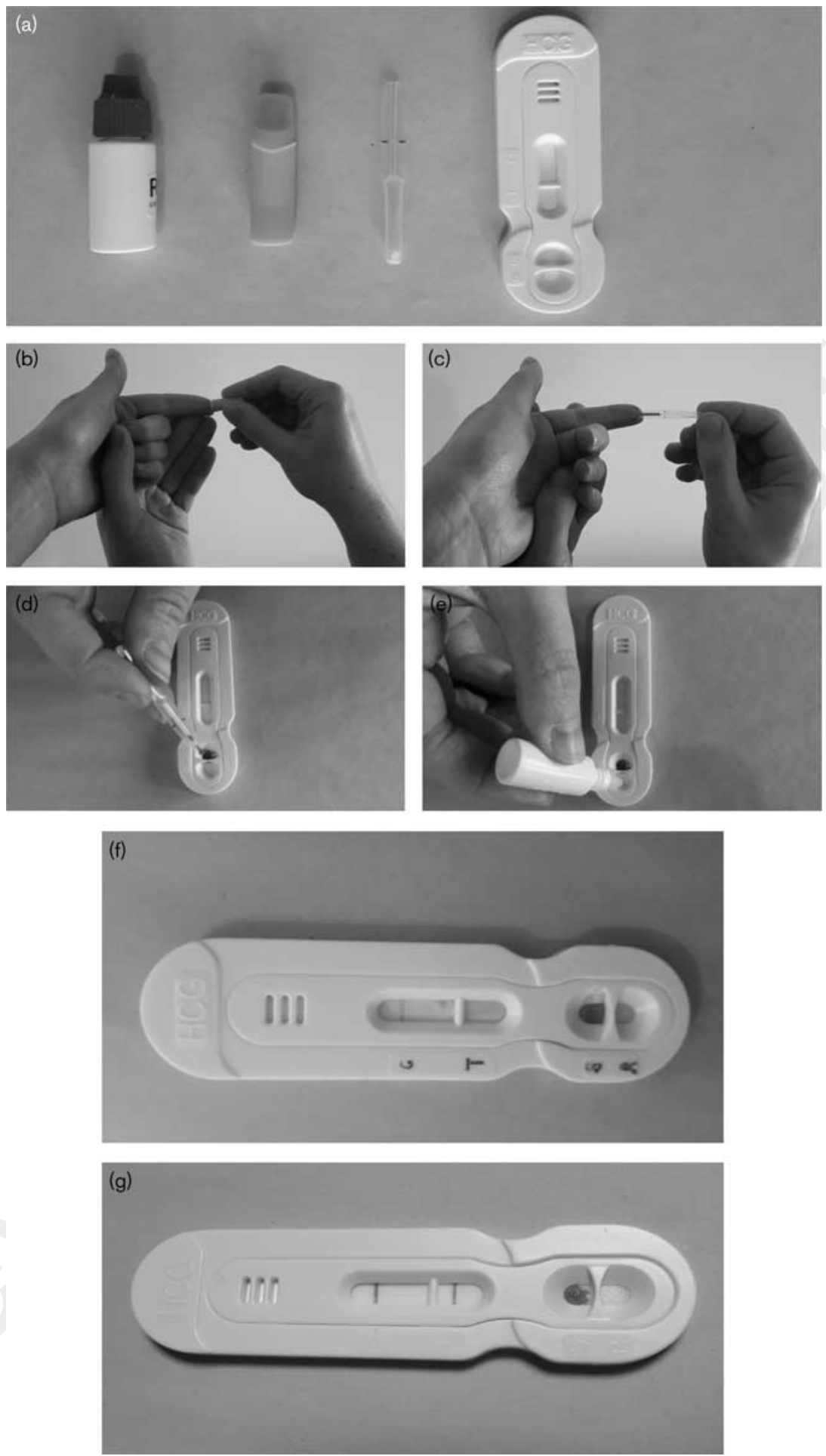

Quick pregnancy test. (a) Test kit. (b) Incision with a lancing device. (c) Sampling with a pipette. (d) Deposition of the blood drop. (e) Addition of reagent. (f) Negative result. (g) Positive result.

Absence of contraception, delayed menstruation, and severe pain (Numerical Pain Scale >6) were significantly more frequent in the pregnant group (Table 1).
Menstruation delay was $9 \pm 12$ days in pregnant patients. In terms of ultrasound findings, the endometrium was significantly thicker $(13.1 \pm 7.3$ vs. $6.2 \pm 4.1, P<0.001)$ and 
Fig. 2

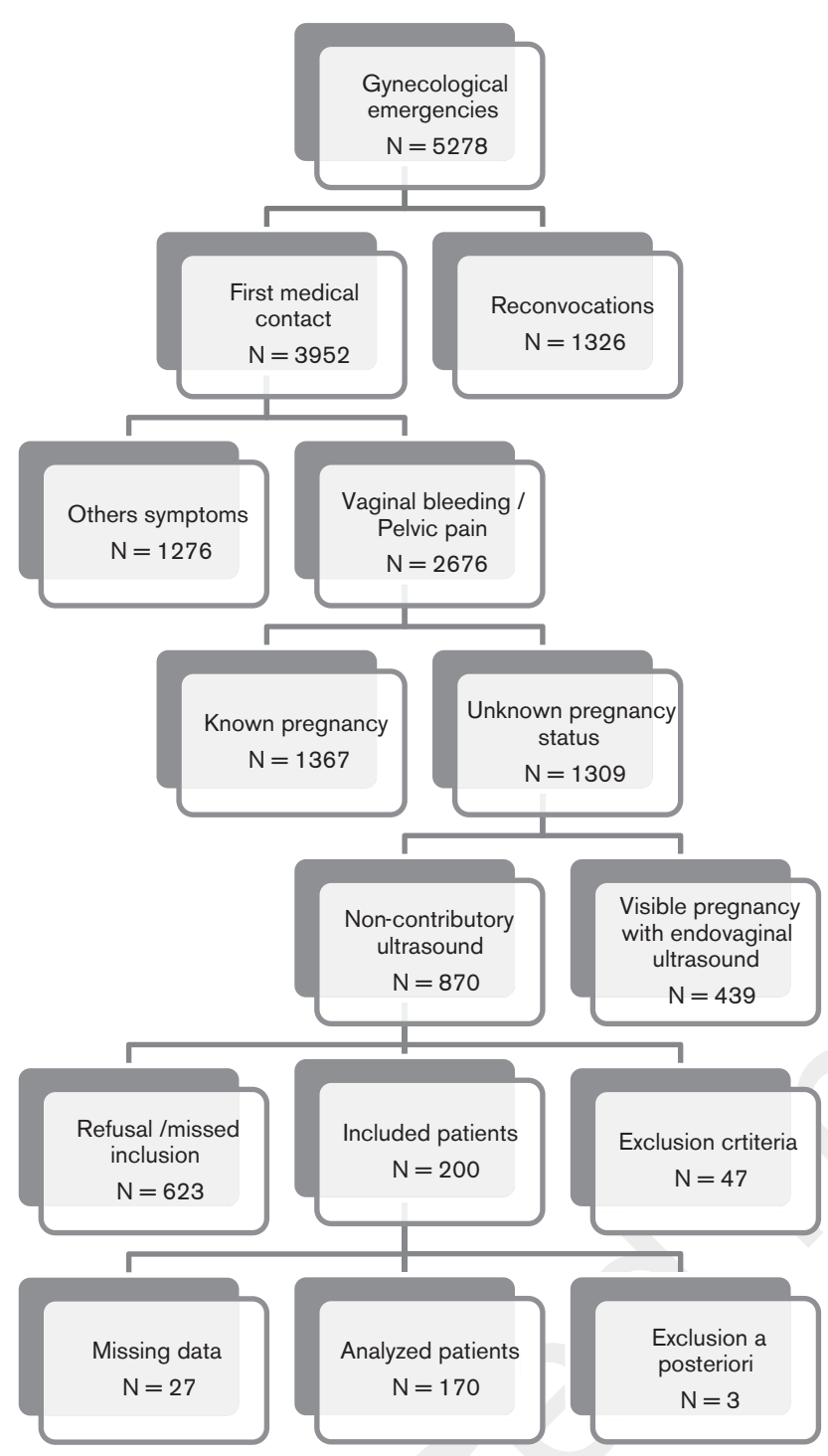

Flow chart.

adnexal masses were significantly more frequent (13.8 vs. $3.5 \%, P=0.047)$ in pregnant women. The results of the quick test, reference test, and final diagnosis are described in Table 2.

\section{NG-Test hCG WB performance}

Twenty-six (15.3\%) patients had a positive NG-Test hCG WB and 29 (17\%) patients were pregnant according to the laboratory test. Sensitivity, specificity, PPV, and NPV of the NG-Test hCG WB were 89.7, 100, 100, and $97.9 \%$, respectively. The Youden index was 0.90 .

Of the three patients with a negative NG-Test hCG WB and a positive laboratory test, two had a quantitative hCG level of $7 \mathrm{UI} / 1$ and the third level was $35 \mathrm{UI} / \mathrm{l}$. The two patients who had a laboratory serum hCG level of $7 \mathrm{UI} / 1$ and a negative NG-Test hCG WB underwent a new laboratory test $48 \mathrm{~h}$ later. The results were negative. These two patients presented with pregnancy and stopped the use of contraceptives. One was consulted for VB and the other for PP and VB. Physical examination and ultrasound findings were normal. The diagnosis of pregnancy was not confirmed. The final diagnoses were menstruation and dysmenorrhea. The third patient, who had a false-negative NG-Test hCG WB with a laboratory serum hCG level of $35 \mathrm{UI} / \mathrm{l}$, had a vacuum aspiration for an early pregnancy loss 1 month ago. The 2-day control showed a negative laboratory test. The retained hypothesis was a residual hCG level following the previous pregnancy.

Using a positivity threshold of $10 \mathrm{IU} / 1$ for the outcome measure, the sensitivity, specificity, PPV, and NPV of the NG-Test hCG WB were 96.3, 100, 100, and 99.3\%, respectively. In this case, the Youden index was 0.96 .

Among the 26 patients presenting a positive pregnancy test for both the NG-Test hCG WB and the laboratory test, there were three patients with EP who had hemoperitoneum requiring immediate surgical management; the other 23 patients had a pregnancy at an unknown location at the time of the initial NG-Test hCG WB. The evaluation indicated 11 IUP, eight early pregnancy losses, and four EP requiring medical treatment with methotrexate.

\section{Quick test use}

Nurses were satisfied with the NG-Test hCG WB at $89 \%$. The test was easy to perform for $56 \%$ of the cases, moderately easy for $26 \%$ of the cases, and difficult for $18 \%$ of the cases. The main difficulty was the obtainment of a sufficient volume of blood. Nurses who had performed the NG-Test hCG WB at least twice did not have any difficulty subsequently. Reading of the results was easy in $99 \%$ of the cases. Reading was considered difficult by the nurse in two cases, for which a second reading was carried out by the doctor, who was unaware of the nurse's answer. Both tests were interpreted positively at both readings. For seven patients, the NG-Test hCG WB had to be performed twice because the first one was not valid. Five control tests were negative and two were positive. This was because of manipulation mistakes during different steps: insufficient waiting time, insufficient reactive quantity, and inversion between the blood reception zone and the reactive zone.

\section{Impact of the quick test on waiting time for patients in the early pregnancy unit}

The mean time spent in early pregnancy units was $254 \pm 78 \mathrm{~min}$ for patients. The mean time between patient arrival and the blood sample taken by the nurse was $121 \pm 58 \mathrm{~min}$. The result of the laboratory test was obtained at a mean time of $90 \pm 31 \mathrm{~min}$. After the biological results, the mean time required to allow patient 


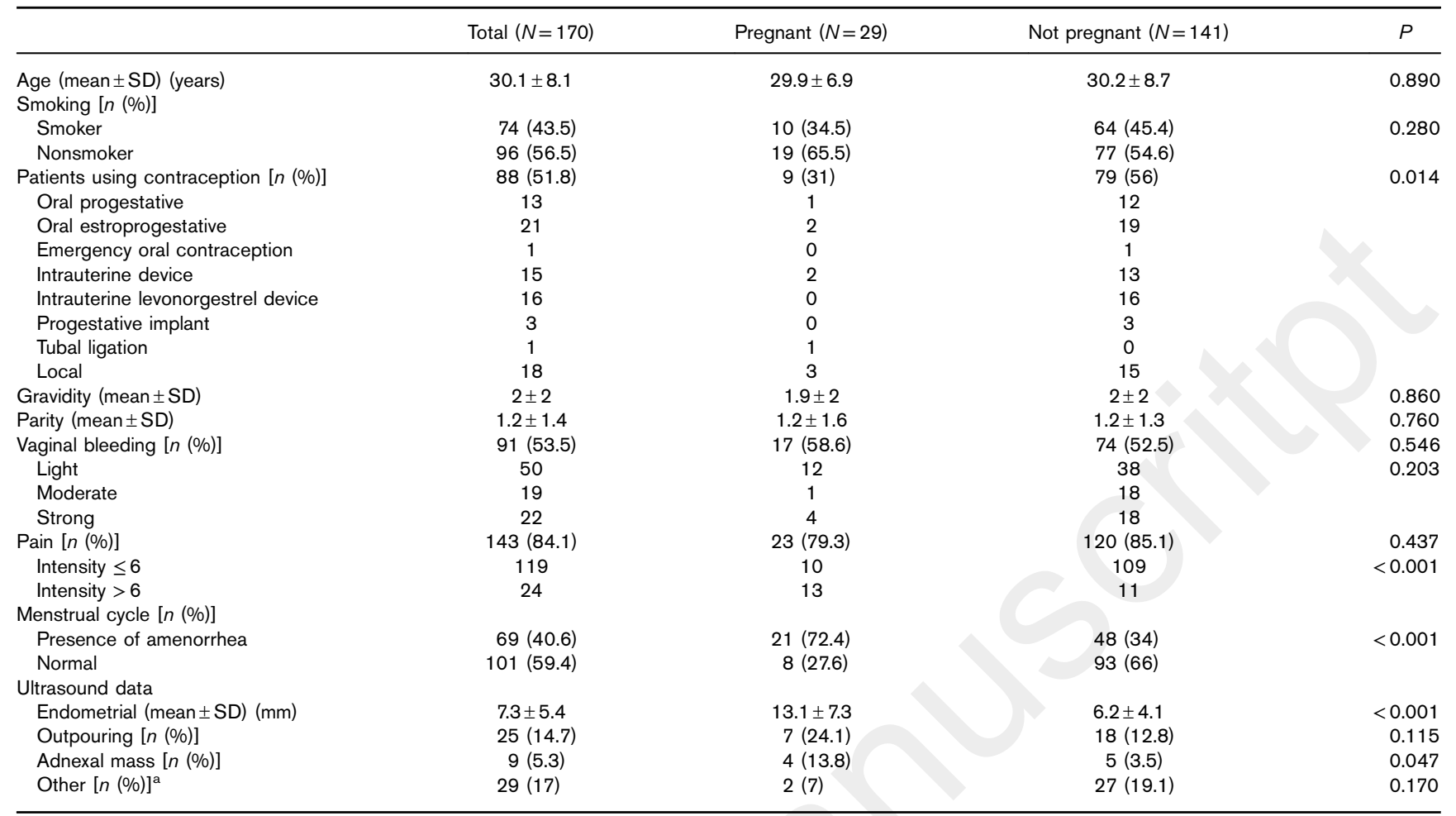

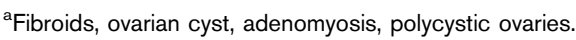

Table 2 Final diagnosis in patients with a positive laboratory test

\begin{tabular}{|c|c|c|c|}
\hline Symptoms & Serum hCG with gold standard test & Quick test & Final diagnosis \\
\hline Vaginal bleeding and pelvic pain & 7 & Negative & Dysmenorrhea \\
\hline Vaginal bleeding & ( & Negative & Menstruation \\
\hline Vaginal bleeding & 17 & Positive & Trophoblastic retention \\
\hline Pelvic pain & 27 & Positive & Intrauterine pregnancy \\
\hline Vaginal bleeding & 35 & Negative & Residual hCG \\
\hline Vaginal bleeding & 101 & Positive & Early miscarriage \\
\hline Vaginal bleeding and pelvic pain & 203 & Positive & Early miscarriage \\
\hline Vaginal bleeding and pelvic pain & 338 & Positive & Early miscarriage \\
\hline Pelvic pain & 339 & Positive & Intrauterine pregnancy \\
\hline Pelvic pain & 349 & Positive & Intrauterine pregnancy \\
\hline Pelvic pain & 353 & Positive & Intrauterine pregnancy \\
\hline Pelvic pain & 464 & Positive & Intrauterine pregnancy \\
\hline Vaginal bleeding & 534 & Positive & Ectopic pregnancy (medical treatment) \\
\hline Pelvic pain & 565 & Positive & IUP \\
\hline Vaginal bleeding and pelvic pain & 823 & Positive & EP (medical treatment) \\
\hline Pelvic pain & 891 & Positive & EP (surgical treatment) \\
\hline Vaginal bleeding and pelvic pain & 1293 & Positive & EP (surgical treatment) \\
\hline Pelvic pain & 1461 & Positive & IUP \\
\hline Pelvic pain & 1624 & Positive & IUP \\
\hline Vaginal bleeding and pelvic pain & 1817 & Positive & IUP \\
\hline Pelvic pain & 2404 & Positive & EP (medical treatment) \\
\hline Vaginal bleeding and pelvic pain & 2700 & Positive & EP (surgical treatment) \\
\hline Vaginal bleeding and pelvic pain & 2747 & Positive & Early miscarriage \\
\hline Vaginal bleeding & 3170 & Positive & Early miscarriage \\
\hline Pelvic pain & 4552 & Positive & IUP \\
\hline Pelvic pain & 4731 & Positive & IUP \\
\hline Vaginal bleeding and pelvic pain & 5939 & Positive & EP (medical treatment) \\
\hline Vaginal bleeding and pelvic pain & 8711 & Positive & Early miscarriage \\
\hline Vaginal bleeding and pelvic pain & 9950 & Positive & Early miscarriage \\
\hline
\end{tabular}

EP, ectopic pregnancy; hCG, human chorionic gonadotropin; IUP, intrauterine pregnancy.

release was $43 \pm 36 \mathrm{~min}$. The time required to obtain the result of the NG-Test hCG WB was 5 min, sparing 85 min compared with the laboratory test.

\section{Cost-effectiveness analysis}

The implementation of the NG-Test hCG WB implies that the laboratory test would be performed only for a 
positive NG-Test hCG WB. The cost of an NG-Test hCG WB is $\sim 4$ euros. In our laboratory, a quantitative plasma dose of hCG costs $\sim 2$ euros, including the reagent and materials needed for the blood sample (compress, needle, tube, etc.). Using the NG-Test hCG WB in our population would have cost 732 euros (170 NG-Test hCG WB + 26 laboratory tests) versus 340 euros for 170 laboratory tests. As a comparison, urine tests cost approximately the same as NG-Test hCG WB.

\section{Discussion}

The NG-Test hCG WB showed good performance in the present study. Indeed, our study showed $89.7 \%$ sensitivity of the quick test with a $5 \mathrm{UI} / \mathrm{l} \mathrm{hCG}$ threshold and $96.3 \%$ sensitivity with a $10 \mathrm{UI} / \mathrm{l}$ hCG threshold.

The patient who had a negative NG-Test hCG WB and a serum hCG level of $35 \mathrm{UI} / \mathrm{l}$ had a residual hCG level after early pregnancy loss, which returned a negative result $48 \mathrm{~h}$ later. Intact hCG is the bioactive form produced by the syncytiotrophoblast and it accounts for the quasitotality of serum hCG in the first trimester [7-9]. The $\beta$ unit is a metabolite arising from its degradation and disappears after intact hCG [9], explaining our falsenegative result.

The two patients who had an hCG level of $7 \mathrm{UI} / \mathrm{l}$ had a negative NG-Test hCG WB, which was expected, given its theoretical positivity threshold. The control was negative and the clinical findings did not suggest a pregnancy. A very low quantity of hCG can be detected in the serum of nonpregnant women, which increased with patient age. Snyder $e$ al. [10] observed hCG levels as high as $7.7 \mathrm{UI} / 1$ before 55 years of age in the absence of pregnancy. According to a European study, $1.1 \%$ of nonpregnant women had hCG levels between 2 and $7 \mathrm{UI} / \mathrm{l}$, which was in agreement with the present study.

However, it is crucial to choose a sufficiently low threshold to detect all pregnancies. The danger is in missing the EPs. The commonly accepted threshold is $5 \mathrm{UI} / \mathrm{l}$ for quantitative tests, keeping in mind that higher levels can be observed in nonpregnant women. For qualitative tests, a $10 \mathrm{UI} / \mathrm{l}$ threshold may be acceptable because the risk of severe complications because of undiagnosed EP with hCG levels between 5 and $10 \mathrm{UI} / 1$ is low. However, the correlation between serum hCG level and the risk of a ruptured EP has been debated [11]. In the case of unstable hemodynamics, both tests could be carried out from the outset, the first allowing a fast and adapted therapeutic management and the second allowing the diagnosis to be rectified without delay if the hCG level is between 5 and $10 \mathrm{IU} / \mathrm{l}$. It is also interesting to note that cases of $\mathrm{EP}$ with hemoperitoneum and negative serum hCG levels have been reported [12,13].

Use of the NG-Test hCG WB reduces the time spent in early pregnancy units. The time required to obtain the pregnancy status results after sampling was reduced from
90 to $5 \mathrm{~min}$. If the result is negative, the information can be dispensed instantaneously and the patient discharged without delay. It has already been established that the use of POC testing reduces length of stay in an emergency department $[14,15]$.

Using the NG-Test hCG WB would increase the cost of pregnancy tests. However, this evaluation did not include the costs of sampling and transport to the laboratory. From our point of view, time savings, optimization of human resources, and optimization of emergency unit throughput could justify the use of the NG-Test hCG $\mathrm{WB}$, although it is currently still investigational.

Some studies evaluated qualitative urinary tests. The level of detection for hCG is $\sim 20-25 \mathrm{UI} / \mathrm{l}$, which is inadequate to detect early pregnancy and leads to falsenegative results [16]. A qualitative whole-blood quick test has several advantages compared with qualitative urinary tests. It reduces the turnaround time for results because there is no need to wait for a spontaneous miction depending on bladder repletion level and patient hydration [17]. The quick test can be performed on anuric patients in the case of hypovolemic shock, a rare but not exceptional situation [18].

The strengths of our study are its prospective setting and the originality of the qualitative whole-blood pregnancy test. It was evaluated in routine clinical practice, and not using a bank blood, to limit bias because of the degradation of hCG. IUP identified with ultrasound were excluded because they do not warrant an indication for a biological pregnancy test. However, this yielded a low rate of positive tests $(16 \%)$ in our population. One limitation was that we did not study the reproducibility or the interobserver variability. No hook effect occurred in our study because it only occurs for higher hCG concentrations (around $500000 \mathrm{UI} / \mathrm{l}$ ) in molar pregnancies or advanced IUP.

\section{Conclusion}

The NG-Test hCG WB using whole blood is effective for diagnosing early pregnancy with a $10 \mathrm{UI} / 1$ positivity threshold. Its use allows a quick triage for patients presenting with VB or PP in the case of noncontributive clinical and ultrasound examinations. Its use for patient follow-up after EP, molar pregnancies, or tumors is not recommended. The use of this diagnosis algorithm would spare human requirements and optimize patient throughput.

\section{Acknowledgements Conflicts of interest}

There are no conflicts of interest.

\section{References}

1 Alouini S, Mesnard L, Coly S, Dolique M, Lemaire B. Gynecological emergencies: etiology and degree of gravity. J Gynecol Obstet Biol Reprod (Paris) 2012; 41:48-54. 
2 Calvo J, Louges MA, Sanchez S, Lipere A, Duminil L, Graesslin O. Gynecological emergencies: a prospective study about patients purpose of consultation and outcome. J Gynecol Obstet Biol Reprod (Paris) 2016; 45:1060-1066.

3 Greene DN. Pathology consultation on human chorionic gonadotropin testing for pregnancy assessment. Am J Clin Pathol 2015; 144:830-836.

4 Woo KM, Director T, Sweeney C, Cristales D, Deguia J, Baumlin K. 417 False negative point-of-care urine pregnancy results in the emergency department: quantifying a needle in the haystack in the clinical setting. Ann Emerg Med 2015; 66:S150.

5 National Institute for Biological Standards and Control. WHO International Standard, 5th WHO IS, chorionic gonadotrophin, NIBSC code: 07/364, instructions for use (version 3.0, dated 06/04/2013) http://www.nibsc.org/ documents/ifu/07-364.pdf.

6 National Institute for Biological Standards and Control. WHO International Standard, 4th IS chorionic gonadotropin, human, NIBSC code: $75 / 589$, instructions for use (version 3.0, dated 30/11/2007) http://www.nibsc.org/ documents/ifu/75-589.pdf.

7 Berger P, Sturgeon C. Pregnancy testing with hCG - future prospects. Trends Endocrinol Metab 2014; 25:637-648.

8 Montagnana M, Trenti T, Aloe R, Cervellin G, Lippi G. Human chorionic gonadotropin in pregnancy diagnostics. Clin Chim Acta 2011; 412:1515-1520.

9 Stenman UH, Tiitinen A, Alfthan H, Valmu L. The classification, functions and clinical use of different isoforms of HCG. Hum Reprod Update 2006; 12:769-784.
10 Snyder JA. Diagnostic considerations in the measurement of human chorionic gonadotropin in aging women. Clin Chem 2005; 51:1830-1835.

11 Downey LV, Zun LS. Indicators of potential for rupture for ectopics seen in the emergency department. J Emerg Trauma Shock 2011; 4:374-377.

12 Grynberg M, Teyssedre J, Andre C, Graesslin O. Rupture of ectopic pregnancy with negative serum beta-hCG leading to hemorrhagic shock. Obstet Gynecol 2009; 113 (Pt 2): 537-539.

13 Lonky NM, Sauer MV. Ectopic pregnancy with shock and undetectable betahuman chorionic gonadotropin. A case report. J Reprod Med 1987; 32:559-560.

14 Singer AJ, Ardise J, Gulla J, Cangro J. Point-of-care testing reduces length of stay in emergency department chest pain patients. Ann Emerg Med 2005; 45:587-591.

15 Asha SE, Chan ACF, Walter E, Kelly PJ, Morton RL, Ajami A, et al. Impact from point-of-care devices on emergency department patient processing times compared with central laboratory testing of blood samples: a randomised controlled trial and cost-effectiveness analysis. Emerg Med J 2014; 31:714-719.

16 Terwijn M, van Schie A, Blankenstein MA, Heijboer AC. Pregnancy detection by quantitative urine hCG analysis: the need for a lower cut-off. Clin Chim Acta Int J Clin Chem 2013; 424:174.

17 Gottlieb M, Wnek K, Moskoff J, Christian E, Bailitz J. Comparison of result times between urine and whole blood point-of-care pregnancy testing. West J Emerg Med 2016; 17:449-453.

18 Habboushe JP, Walker G. Novel use of a urine pregnancy test using whole blood. Am J Emerg Med 2011; 29:840.e3-840.e4. 